Background: The coronary and cerebral perfusion in patients with sudden cardiac arrest in prehospital conditions mostly depends on quality of chest compression taken by the witness, and on the medical emergency procedures implemented by ambulance teams. Methods: The retrospective studies analyzed 1,078 cases of a sudden cardiac arrest that occurred in the Siedlce District, Poland. The collected data emerged from medical documentation of Emergency Ambulance Service in Siedlce. The influence of early BLS implementation by witnesses before ambulance arrival, the type of ambulance medical staff performing medical actions, and ambulance arrival time to a place of an accident were taken under consideration on the effectiveness of a successful cardiopulmonary resuscitation. If the return of spontaneous circulation was obtained and a patient was taken to hospital, actions were considered to be effective.

Results: In the years 2013, 2014, and 2015, there were respectively reported 345,354 , and 379 cases of a sudden cardiac arrest. Having analyzed the whole group of 1,078 cases - early BLS implementation before ambulance arrival was taken by $31 \%$ of witnesses. It was noticed that taking early BLS implementation significantly increased the effectiveness of a resuscitation from $31 \%$ to $53 \%$. Ambulance arrival time was compared (BLS/non-BLS cases) and it was 7 minutes 51 seconds and 8 minutes 12 seconds, respectfully. Moreover, the type of ambulance medical staff (with and without a doctor) did not have any impact on the effectiveness of a resuscitation. Conclusion: The effectiveness of emergency medical actions, in cases of a sudden cardiac arrest especially, depends on early implementation of BLS by witnesses in prehospital condition. Prehosp Disaster Med 2017;32(Suppl. 1):s173-s174

doi:10.1017/S1049023X17004642

\section{Does EMS Performance Lead to a Reduced Number of} Organ Donors?

Markku Kuisma ${ }^{1}$, Mari Murro ${ }^{2}$, Ari Salo ${ }^{1}$, James Boyd ${ }^{1}$

1. Section Of Emergency Medical Services, Helsinki University Hospital and University of Helsinki, Helsinki/Finland

2. Department Of Perioperative, Intensive Care And Pain Medicine, HUCH, Helsinki/Finland

Study/Objective: To evaluate whether Emergency Medical Service (EMS) identifies potential organ donor candidates and initiates the care needed.

Background: According to Finnish Organ Donation guidelines, all patients in need of organ transplantation should have timely and fair access to transplants. Thus, every potential donor should be identified and referred to intensive care. For most potential donors, EMS is the point of entry to medical care, and unconsciousness is the most typical presentation in the prehospital setting.

Methods: We conducted a retrospective study with data from Helsinki EMS hospital and out-of-hospital cardiac arrest databases. Patients included were $\leq 80$ years old, with a Glasgow Coma Score $\leq 8$, and transported to hospital with code "702" (unconscious) in 2015, and EMS witnessed traumatic cardiac arrests in 2013-2015.

Results: In 2015, we identified 84 patients belonging to this study group; 24 patients (male $\mathrm{n}=12$, median age
$57[\mathrm{IQR}=48-66]$ years) were later diagnosed with stroke, traumatic brain injury, or were resuscitated from cardiac arrest and did not have contraindications for organ donation in theory. Of those, 22 patients were intubated and ventilated on scene, of which 11 died later in the hospital. Six of them were considered for organ donation and two patients became organ donors. Both patients who were not intubated on scene, and allowed to breath spontaneously, were subsequently intubated in the emergency department and considered for organ donation, and one of them became an organ donor. In 2013-2015, four trauma patients had an EMS witnessed cardiac arrest (male $\mathrm{n}=3$, median age 32 years). Three of them received Basic Life Support cardiopulmonary resuscitation. All had clinical evidence of severe blunt multitrauma and they died on scene.

Conclusion: Possible organ donor candidates are well recognized in EMS. EMS performance does not lead to a reduced number of organ donors.

Prehosp Disaster Med 2017;32(Suppl. 1):s174

doi:10.1017/S1049023X17004654

\section{Cold Exposure After a Train Crash - An Experiment in a Cold Environment Jonas Aléx, Rebecca Forsberg}

Research And Development Center For Disaster Medicine, Unit of Surgery, Department Of Surgical And Perioperative Science, Umeå University, Umeå/Sweden

Study/Objective: To explore the air cooling rate in an intact rail carriage, in a cold environment, after power was turned off.

Background: There have been a growing number of train disasters globally. The high speeds increase the risk of severe injuries, and many train routes are far from navigable roads. Rescue operations after international train crashes have been complicated and lengthy. In the circumpolar regions, cold exposure has to be added as a negative factor, as hypothermia could be lethal, especially in combination with trauma. However, there is a lack of knowledge about the specific milieu that the passengers could be exposed to in a train crash in a cold environment.

Methods: The experiment was performed inside an intact train, type Coradia Nordic X62, in Sweden. The outdoor temperature was $-13.8^{\circ} \mathrm{C}$, and inside the carriage the air temperature at the floor was $+21.0^{\circ} \mathrm{C}$ when the power was cut. Air temperature was measured by air loggers every 30 seconds. Results: After one hour, the air temperature at the floor reached approximately $15^{\circ} \mathrm{C}$, after two hours, $12^{\circ} \mathrm{C}$, and after four hours, $6^{\circ} \mathrm{C}$. A theoretical simulation shows that the floor temperature should reach temperatures below zero after seven hours.

Conclusion: The results draw attention to the importance of a rapid rescue operation, as well as an ability to retain heat in the carriages, is of great importance in order to reduce the risk for secondary injuries and fatalities due to hypothermia. In order to fulfill the needs of thermal comfort for passengers travelling in cold environments, and in rural areas far from passable roads, more effective material not dependent on electricity might be needed in the trains.

Prehosp Disaster Med 2017;32(Suppl. 1):s174

doi:10.1017/S1049023X17004666 